\title{
Large-area Floating Strip Micromegas
}

\author{
Jonathan Bortfeldt*; Otmar Biebel, Ralf Hertenberger, Philipp Lösel, Samuel Moll \\ and André Zibell \\ LS Schaile, Ludwig-Maximilians-University Munich \\ E-mail: jonathan.bortfeldt@ecern.ch
}

\begin{abstract}
Micromegas (MICRO MEsh GAS detectors) are high-rate capable, high resolution micro pattern gas detectors. Resistive strip Micromegas with square meters in area are foreseen as replacement of the precision chambers in the small wheel region of the ATLAS muon spectrometer. The resistive anode material in the active area diminishes amplitude and duration of discharges.

As a novel approach a Micromegas with floating strip anode and an active area of $50 \mathrm{~cm} \mathrm{x} 48 \mathrm{~cm}$ has been developed. The anode consists of 1920 copper strips that are individually connected to high-voltage and can float in potential during a discharge. Thus the area affected by a discharge can be strongly reduced. Measurements with high-energy pion beams at SPS/CERN have been performed. We discuss the observed efficiency, pulse height behavior and spatial resolution. The substantial reduction of dead time after discharges due to the floating strip principle is demonstrated and compared to results from an LTSpice detector simulation.
\end{abstract}

The European Physical Society Conference on High Energy Physics -EPS-HEP2013

18-24 July 2013

Stockholm, Sweden

\footnotetext{
* Speaker.

†This work was supported by the DFG cluster of excellence on "Origin and Structure of the Universe" and the DFG research training group 1054.
} 


\section{Introduction}

Micromegas are microstructured gaseous detectors, introduced in 1996 [1], that are high-rate capable by exploiting short ion drift times due to gas amplification between a fine micro mesh and anode strips. Due to the elevated background hit rates in the Small Wheel Region of the ATLAS muon spectrometer after the LHC luminosity upgrade to $2-5 \times 10^{34} \mathrm{~cm}^{-2} \mathrm{~s}^{-1}$, Micromegas have been chosen as replacement for the currently used Monitored Drift Tube and Cathode Strip precision tracking chambers [2].

A challenge when operating Micromegas detectors are non-destructive discharges between the micro mesh and the anode strips. They are induced by charge densities exceeding $10^{8} \mathrm{e} / \mathrm{mm}^{2}$ which are created by strongly ionizing particles and create dead time due to the necessary recharge of the mesh or the anode strips respectively. Since the discharge forms a well conducting plasma channel the potential between the mesh and all affected readout strips equalizes. The efficiency as well as the spatial resolution can be degraded.

We present the performance of novel floating strip Micromegas detectors with enhanced discharge protection. We commissioned and investigated a $50 \mathrm{~cm} \times 48 \mathrm{~cm}$ floating strip Micromegas with 1920 strips. A similar approach, although not quite as radical with respect to resistor and capacitor values, has been proposed by [3]. Copper anode strips, with a width of $150 \mu \mathrm{m}$ and a pitch of $250 \mu \mathrm{m}$ are individually connected to positive high-voltage of $500 \mathrm{~V}$ via $22 \mathrm{M} \Omega$ resistors, whereas the micro mesh is held at ground potential (Figure 1). Small high-voltage capacitors are used to decouple the signal from the strips. Their capacitance is of the same order as the stripto-mesh-, strip-to-strip- and strip-to-ground-capacitance. Although this leads to a loss of signal, it significantly improves the discharge behavior: Since the discharge is localized, only few strips are affected in a discharge. This has two advantages compared to the standard, non-resistive Micromegas configuration, where the mesh would discharge completely onto the strips. First, since the capacitance of the discharging strips is small compared to the capacitance of the other strips, the global voltage drop on the common strip potential is negligibly small. The rest of the detector stays unaffected by the discharge. Second, the small strip capacitance considerably reduces the recharge time.

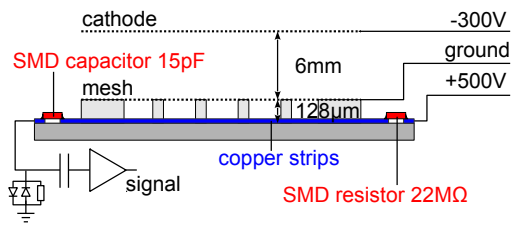

Figure 1: Schematic setup of a floating strip Micromegas.

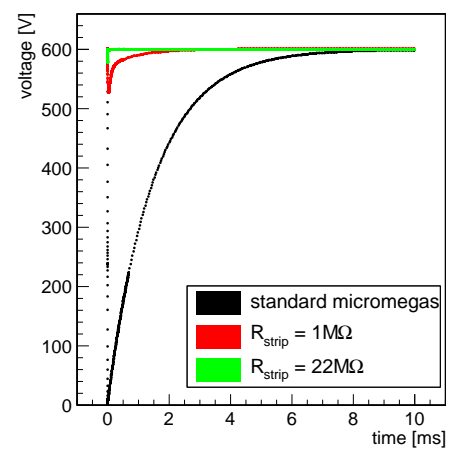

Figure 2: Simulated global voltage drop for different recharge resistors.

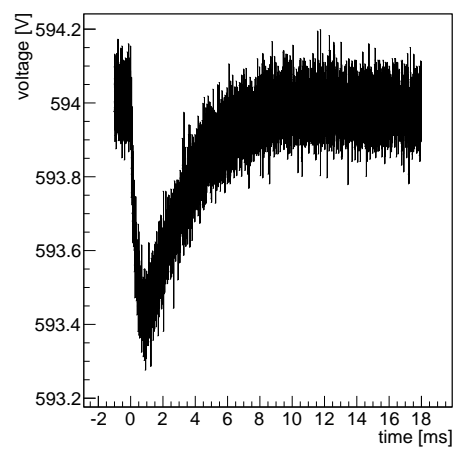

Figure 3: Measured global voltage drop with a recharge resistor of $22 \mathrm{M} \Omega$. 


\section{Performance}

The floating strip Micromegas, operated with an $\mathrm{Ar}: \mathrm{CO}_{2}$ 93:7 \%vol gas mixture, has been investigated with $120 \mathrm{GeV}$ pion beams at the SPS/CERN. A telescope, consisting of six non-resistive Micromegas with one-dimensional readout and two resistive Micromegas with two-dimensional readout was used as track reference for the large floating strip Micromegas [4]. The detector response has been studied for different beam position along and perpendicular to the strips, for detector inclinations between $0^{\circ}$ and $40^{\circ}$, for different gas pressures and particle rates as a function of the amplification and drift voltage. We observe efficiencies of $(95 \pm 2) \%$, an optimum spatial resolution of $(48 \pm 2) \mu \mathrm{m}$ and a homogenous pulse height with only $15 \%$ variation over the detector. By recording the arrival time of single charge clusters on readout strips for inclined tracks, the track inclination can be determined directly from a single readout plane. For inclined tracks between $15^{\circ}$ and $35^{\circ}$ a single plane angular resolution of $5^{\circ}$ has been measured.

In order to demonstrate the discharge suppression ability, a dedicated floating strip Micromegas with 128 readout strips and an active area of $64 \mathrm{~mm} \times 64 \mathrm{~mm}$ has been constructed. Exchangeable coupling capacitors and recharge resistors allowed for the optimization of the detector. A simplified Micromegas model, considering the strip-to-mesh-, strip-to-strip- and strip-to-ground-capacitances in the detector, has been investigated with the circuit simulation program LTSpice. As can be seen from Figure 2, the global high-voltage drop after a discharge is smallest for a recharge resistor of $22 \mathrm{M} \Omega$. This value has been chosen as compromise between low global voltage drop and sufficiently fast strip recharge time. The measured mean global voltage drop is then $0.6 \mathrm{~V}$, which is negligible (Figure 3).

\section{Conclusion}

We commissioned and investigated a floating strip Micromegas with novel discharge protection anode. The detector has been tested in $120 \mathrm{GeV}$ pion beams and showed high efficiency of $(95 \pm 2) \%$, good spatial resolution of $(48 \pm 2) \mu \mathrm{m}$ and a homogeneous pulse height. We could show, that the mean voltage drop of the common amplification voltage after a discharge is only $(0.6 \pm 0.1) \mathrm{V}$ and that the recharge is fast. This is in qualitative agreement with a detector simulation in LTSpice. Floating strip Micromegas are currently tested for medical applications in protonand carbon-beams with particle rates between $2 \cdot 10^{6} \mathrm{~Hz}$ and $2 \cdot 10^{9} \mathrm{~Hz}$.

\section{References}

[1] Y. Giomataris, P. Rebourgeard, J. Robert and G. Charpak, Micromegas: a high-granularity position sensitive gaseous detector for high particle-flux environments, Nucl. Instr. and Meth. A 376 (1996) 29 35.

[2] ATLAS Collaboration, New Small Wheel technical design report, CERN/LHCC 2013-006 (2013).

[3] A. Bay, J. P. Perroud, F. Ronga, J. Derré, Y. Giomataris, A. Delbart and Y. Papadopoulos, Study of sparking in Micromegas chambers, Nucl. Instr. and Meth. A 488 (2002), no. 1-2 162 - 174.

[4] J. Bortfeldt, O. Biebel, R. Hertenberger, A. Ruschke, N. Tyler and A. Zibell, High-resolution Micromegas telescope for pion- and muon-tracking, Nucl. Instr. and Meth. A 718 (2013), no. $0406-$ 408. 